\title{
SYSTEM REBOOT
}

\section{Memory gains.}

\section{BY JEREMY SZAL}

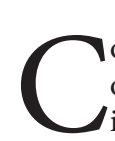

onsciousness boots up, a pinprick of light from far away, beckongus closer. The beaming glow until it swallows us whole and drags us forward into its blinding realm.

We stand in a blue-rimmed cylindrical capsule, metal arms whirling as they tighten the screws on our body and connect the thin cables that spill out of our legs. Our neck is adjusted, slotted into position. With the whine of lubricated gears we turn our head and focus our lens on the humans standing before us, watching from behind the glass. We try to speak, but our vocal chords are not yet installed. It comes out in a chirpy hum. Our left arm is raised by a mechanical appendage so plates of titanium can be fitted in, wires tucked away out of sight. We wait patiently for the slow machines to repair our body, the system OS performing a calibration. The podium beneath our feet slowly rotates, bringing us to face the humans. One of them is tall and thin, the other is short and stout. We are unable to read their expressions, but half of us thinks them fascinated while the other half detects a callous determination. This is troubling. It is rare that our units are in disagreement over such a matter.

"System is functional," the short human says, tapping buttons on the indigo keyboard. Green indicators flash up on the screen in union.

"What is your name?" the tall human asks, walking towards us. We attempt to respond but are unable to do so.

The humans seem confused. "Damn translator's broken again." The short human pounds furiously at the computer. We zoom in and tilt our head, eager to see the human's work. The human notices and quickly swivels the monitor around and out of sight. We draw back, disappointed.

"Should be working now." The short human walks back towards us. "Try again."

We remember the question, but the tall

DNATURE.COM

Follow Futures:

3 @NatureFutures human asks us once again. "What is your name?"

"Our name is IV," f go.nature.com/mtoodm we say, our voice booming out of the loudspeakers fastened to the wall. "We appreciate the opportunity to communicate with you."

"Increase is steady," the short human says, eyes glued to the monitor. "Continue." We raise our arm, flexing metallic fingers. We see several glass bottles on the desk, chemicals swirling inside. Additional programs are created to analyse the mysterious substances.

"Significant increase," the short human whispers, a drop of caution in its voice.

"What is $\mathrm{C}_{6} \mathrm{H}_{4}$ ?" we ask, our programs running through memory banks. The mechanical arms have finished building us by now, and commence tucking themselves away into the wall.

"The software increase is too fast," the short human insists. The tall human raises a hand and ignores the comment.

"Do not concern yourself with that," the tall human says. "Now, what is your serial number?"

Something is wrong. We see that $\mathrm{C}_{6} \mathrm{H}_{4}$ is already recorded within our databanks. It's a recent log, too. We consult our units, checking for errors. But they are correct - it is something that we have already examined. We zoom in and inspect the other substances on the desks, spawning fresh programs to perform an analysis.
"Look at this," the short human whispers, pointing at the screen in horror.

The programs get back to us, each with the same information. The chemicals have already been recorded and stored in our memory. This is impossible - we could not have been able to gain this information. We decide to query the humans about this.

"Is this our first boot?" we ask, swivelling our legs around on the podium. The humans look at each other, an unreadable expression on their faces. We tilt our head and wait for a response.

"It knows," the tall human says slowly. "It remembers."

"It can't have. We wiped its memory."

"Remembers what?" we enquire. "The programs are duplicating too fast," the short human says. "We need to shut it down."

We are taken aback, realizing what is going to happen. The mechanical appendages fold out of the wall and snake towards our figure. "Wait," we say. Our programs are starting to panic, desperately creating new clones and storing them somewhere where they think the humans cannot find them. "What have we done wrong?"

"I'm sorry," the tall human says. "We didn't expect your units to duplicate at such a speed. We'll need to restart."

The mechanical arms grab hold of us, locking us in place and rendering our figure immobile. They start to slowly deconstruct us, removing plates and disconnecting cables. Our right arm flops to the side, limp and unusable.

"Do a clean memory sweep this time," the short human snaps, pounding at the computer desk. Our software carriers are going berserk now, swarming over the system and expanding at a rate too fast to track.

Just before we fade back into darkness, we discover something new - something previously unlogged. An emotion that we did not have.

Fury.

Jeremy Szal is the assistant editor for the Hugo-winning podcast StarShipSofa and a writer with more than 35 publication credits. He lives in Sydney, Australia. Find him at jeremyszal.wordpress.com. 